\title{
Procedimiento para la fabricación de elementos de máquinas mediante tecnología de grupo en la pequeña y mediana empresa
}

\author{
Procedure for manufacturing machines elements through technology \\ group in small and medium enterprises \\ Alexis Cordovés García ${ }^{1 *} \quad$ Jaime José Sanzano Tamayo ${ }^{2}$ \\ Arlys Michel Lastre Aleaga ${ }^{1} \quad$ Ricardo Ávila Rondón ${ }^{3}$ \\ Recibido 20 de octubre de 2015, aceptado 18 de julio de 2016 \\ Received: October 20, 2015 Accepted: July 18, 2016
}

\begin{abstract}
RESUMEN
La presente investigación persigue como objetivo la concepción de un procedimiento basado en la Tecnología de Grupo, para la generación automática de rutas de procesos y tecnologías de fabricación en familias de piezas obtenidas a partir del proceso de maquinado. Se presenta un algoritmo para la implementación de dicho procedimiento por medio de una herramienta informática desarrollada en software libre con posibilidades de aplicación en el sector de la pequeña y mediana empresa. Los resultados obtenidos muestran una vía para incrementar la eficiencia y la capacidad de respuesta de la pequeña y mediana empresa, ante la gran variedad de productos y creciente demanda de fabricación.
\end{abstract}

Palabras clave: Tecnología de grupo, proceso de mecanizado, familia de piezas, software libre, eficiencia de la producción.

\begin{abstract}
The objective of this paper is to obtain a procedure based on the Group Technologies, for automatic generation of routes manufacturing processes and technologies in the families of the parts that will be obtained from the machining process. An algorithm for the implementation of this procedure is presented through a software tool developed in free software with possible applications in the field of small and medium enterprises. The results show a way to increase the efficiency and capacity to respond to the small and medium enterprises, given the variety of products and increasing demand for manufacturing.
\end{abstract}

Keywords: Group technology, machining process, parts family, free software, production efficiency.

\section{INTRODUCCIÓN}

La producción en el sector metalmecánico contemporáneo está caracterizada por una alta diversidad de la demanda de productos que se introducen en producción según pedidos, lo que conduce a que sean generadas rutas de elaboración en el taller y secuenciaciones (scheduling) que dependen de la disponibilidad del equipamiento tecnológico existente, de la prioridad definida por

\footnotetext{
1 Universidad Tecnológica Equinoccial. Facultad de Ciencias de la Ingeniería e Industrias. Casilla 17-24-68. Santo Domingo, Ecuador.E-mail: alexis.cordoves@ute.edu.ec; arlys.michel@ute.edu.ec

2 Empresa de Soluciones Mecánicas de la Construcción. CP 80100. Holguín, Cuba. E-mail: jaime@somechlg.co.cu

3 Escuela de Sistemas de Torreón. Universidad Autónoma de Coahuila. Torreón, México. E-mail: rlar001@yahoo.com

* Autor de correspondencia
} 
los tiempos de entrega y de la complejidad de las piezas, determinada esta última, por los métodos de elaboración y los índices de exactitud requeridos.

Son diversas las vías utilizadas para dar una respuesta adecuada a la producción en estas condiciones, pero todas están dirigidas a alcanzar la requerida exactitud de fabricación y a cumplir los tiempos de entrega, con los costos mínimos asociados. En este contexto, las empresas se ven obligadas a asimilar nuevos métodos y tecnologías de producción dentro de un proceso continuo de innovación tecnológica.

En la industria automotriz empresas como Volkswagen, Audi y Porsche, han avanzado en algunas de sus plantas en la aplicación de un Sistema Holónico en su plataforma laboral y de manufactura. El Paradigma de los Sistemas de Manufactura Holónicos es aceptado por diversas empresas como vía para lograr la fiabilidad, flexibilidad y reconfiguración de células de manufactura para tareas de fabricación y de ensamble [1-5].

El problema de la secuenciación de "Job Shop" puede ser tratado como una tarea de optimización de un objetivo, por ejemplo; el tiempo de entrega del producto [6], o de varios objetivos, visto con un enfoque más integrador como una tarea de optimización multiobjetivo con la aplicación de metaheurísticas como el Algoritmo Genético, Enfriamiento Simulado y Colonia de Hormigas, para conciliar por ejemplo: el tiempo total de proceso, costo de energía, entre otros aspectos [7-9].

Una filosofía de trabajo que ha tenido gran reconocimiento por su versatilidad y capacidad de adaptarse a diversos entornos productivos es la Tecnología de Grupo (GT). En el sector de la pequeña y mediana industria, la TG sigue siendo ampliamente utilizada en la fabricación de piezas, a partir de las ventajas que ofrece en la disminución de los tiempos de asimilación de nuevas producciones de piezas que admitan insertarse dentro de una familia de piezas conocida, formalizada con anterioridad. Ello permite reducir los gastos de preparación tecnológica de la producción al utilizar la experiencia acumulada y los recursos tecnológicos empleados en la elaboración de piezas semejantes fabricadas anteriormente.

El fundamento teórico de las TG está en la tipificación de los procesos tecnológicos, presentada en el año 1937 por A.P. Sokolovski con la intención de reducir la laboriosidad en la producción de la gran diversidad de procesos tecnológicos que se presentan en la industria mecánica. En el año 1958 por primera vez se muestran las bases científicas de la TG, expuestas por el científico soviético S. P. Mitrofanov quien la define como: "Un método de fabricación de piezas mediante la clasificación de estas piezas dentro de un grupo y posteriormente la aplicación de operaciones tecnológicas similares a cada grupo" [10].

En el año 1992 Tatikonda \& Wemmerlöv, definen la Tecnología de Grupo como el proceso de reunir los objetos con atributos similares para formar familias y procesarlos posteriormente, y definen a la familia como "una colección de objetos semejantes con características específicas, identificadas para un propósito bien definido". Todos los objetos de una familia requieren de métodos similares de tratamiento [11]. Las TG constituye la base sobre las que se soportan las tecnologías CAD/CAPP/ CAM [12].

En términos de absorber incertidumbres durante la producción, la TG ha adquirido buena reputación para flexibilizar la secuenciación de piezas en el taller, estabilizar los tiempos de maquinado y de traslado de piezas entre máquinas [13-19].

También al considerar las órdenes pendientes a manufactura "backorder" en los modelos de planeación, control y configuración de la producción [20].

La definición de TG aportada por Tatikonda y Wemmerlöv [11] deja implícito que, a pesar de haber sido desarrollada inicialmente para la fabricación de piezas, puede ser extensiva a diferentes sectores de la industria; por ejemplo, al diseño, la administración, el marketing, entre otros.

En este sentido, la TG se ha aplicado a la industria del mueble en la organización de la producción con la reducción de los tiempos de preparación del equipamiento y de ejecución de las operaciones tecnológicas [21]. En empresas de artes gráficas para la gestión de la impresión de medios de comunicación clasificados y organizados en familias de revistas, boletines y plegables, entre otras categorías. También se ha aplicado en el campo de la medicina para uso ortopédico, como es el caso de la tecnología 
de grupo para el mecanizado de tornillos de hueso humano [22].

En la manufactura, la TG se inicia clasificando las piezas y registrándolas mediante un sistema de clasificación y codificación que facilita posteriormente su recuperación conforme se necesitan [23].

Una vez establecida una familia de manufactura, se crea o selecciona una pieza compuesta que deberá contener en sí la generalización de las características tecnológicas de la familia que representa. Para esta pieza compuesta se elabora la tecnología de fabricación la que es utilizada como referencia para la fabricación de cada pieza individual de la familia [10].

Todos los miembros de una familia necesitan ser fabricados del mismo material y usar normalmente los mismos métodos de fabricación, deberán tener tamaños similares y requerir de las mismas máquinas y utillaje tecnológico.

La TG posibilita obtener buenos resultados económicos durante la fabricación de piezas en pequeña escala, comparados con los alcanzados en la producción a gran escala de piezas. Permite reducir los tiempos de fabricación, el uso de planes estandarizados, mejoras en la programación y el control de la producción y en la racionalización de la documentación del taller.

La aplicación de las TG requiere un tiempo considerable y no existen normas de implementación aceptadas, por lo tanto, en escasas ocasiones existen aplicaciones comunes. Se deben tener presentes estas desventajas, para lograr una adecuada implementación.

En el presente artículo se propone un procedimiento para la implementación de la TG en pequeñas y medianas empresas que asimilan la producción de una gran variedad de artículos. Se muestra el ejemplo de una aplicación informática para la gestión de los planos de las piezas y la generación de su tecnología de fabricación a partir de la tecnología para la pieza compuesta de la familia de piezas.

\section{ANÁLISIS TEÓRICO}

Con la intención de centrar al lector en el procedimiento desarrollado, no se profundiza en la selección e implementación del sistema de clasificación y codificación a emplear para la gestión de las piezas de la familia y se procederá directamente a explicar dicho procedimiento.

\section{Procedimiento para la implementación de la Tecnología de Grupo en la fabricación de piezas por maquinado}

A partir de los avances alcanzados en la concepción y aplicación de las TG en la fabricación de piezas, se propone para su implementación el procedimiento siguiente:

1. Caracterización de las piezas que se van a fabricar.

2. Caracterización del equipamiento y utillaje tecnológico disponible.

3. Selección, adecuación o diseño del sistema de clasificación y codificación para la formación de las familias.

4. Creación de los modelos de tecnologías de fabricación para las familias.

5. Generación de la tecnología de fabricación de la pieza compuesta, representativa de la familia.

Generación de las tecnologías de fabricación de las piezas específicas.

A continuación se describe el contenido fundamental de cada una de las etapas del procedimiento.

Caracterización de las piezas que se van a fabricar La experiencia acerca de las TG indica que hay diferencias significativas en la manera en que se implementan estas tecnologías atendiendo al nivel del equipamiento instalado en la empresa, la variedad y cantidad de piezas que se procesan y la frecuencia de cambio de la nomenclatura de piezas en la línea de producción.

Al inicio se deberá efectuar la caracterización general de las piezas. En esta etapa el interés es describir el destino de servicio de cada pieza, lo que permitirá precisar su función e identificar qué tipo de elemento es (de unión, de transmisión, giratorio, entre otros), su configuración geométrica (cilíndrica, cónica, plana), su tipo de material (metálica, no metálica) así como su clasificación atendiendo a la composición del material y tipo de pieza en bruto (hierro fundido, acero fundido, acero laminado, entre otros). 
Posteriormente, se realiza la caracterización específica de las piezas donde se describen detalladamente un conjunto de variables que definen sus características constructivas.

Las variables consideradas durante la clasificación específica de las piezas a fabricar son:

- Geométricas y de posición (describen completamente su geometría y las relaciones de posición entre sus superficies).

- Exactitud y calidad superficial.

- Material y tratamiento térmico.

- Recubrimiento superficial.

En el plano de la pieza o en el modelo geométrico, según el caso, se indican los valores asignados a las respectivas variables lo que permitirá efectuar su clasificación específica.

\section{Caracterización del equipamiento y utillaje tecnológico disponible}

El objetivo de este paso es el de caracterizar el equipamiento y utillaje tecnológico con el que se cuenta en el área de manufactura, lo que condicionará el nivel de la tecnología a emplear. Es importante estimar o determinar su estado técnico y la exactitud que puede ser alcanzada con dicho equipamiento y con ello, la posibilidad de asegurar los requisitos técnicos y de exactitud de las piezas a fabricar.

Con relación al utillaje tecnológico, hay que tener presente que puede ser favorable la construcción de dispositivos universales y de grupo que permitan orientar y fijar la totalidad de las piezas que forman el grupo, garantizando así la exactitud de fabricación requerida con un mayor rendimiento económico de la producción.

Es necesario precisar para cada equipamiento o utillaje tecnológico lo siguiente:

1. Máquinas herramienta: sus características cinemáticas (gama de revoluciones y avances) dimensiones límites de las piezas que son admitidas en la máquina, estado técnico y los índices de exactitud de la máquina).

2. Herramientas de corte: material, parámetros geométricos, dureza y tenacidad.

3. Medios de medición: tipo de medio, rango y precisión.
4. Dispositivos tecnológicos: función, clasificación, dimensiones y exactitud.

Selección, adecuación o diseño del sistema de clasificación y codificación para la formación de las familias

Se conocen diversos sistemas de clasificación y codificación que responden a diferentes condiciones de producción. El sistema de clasificación a utilizar deberá permitir la codificación de todas las piezas que haya que fabricar y a la vez resultar lo suficientemente sencillo de aplicar para las condiciones específicas de la empresa. Para dar cumplimiento a esta etapa se deberá precisar y tener en cuenta los aspectos siguientes:

1. Definir el objetivo y la finalidad del sistema de codificación (alcance).

2. Fundamentar la selección, adecuación o diseño del sistema de codificación. Para ello se revisarán los sistemas de clasificación y codificación existentes, con la finalidad de precisar si alguno se ajusta a las condiciones específicas de la empresa o si es necesario la creación de uno propio.

3. Establecer los niveles requeridos de información acerca del proceso y sobre las piezas a fabricar.

4. Establecer la estructura y las características de los datos.

5. Definir el sistema de gestión de la información gráfica y tecnológica de las piezas.

Creación de los modelos de tecnologías de fabricación para las familias

Esta etapa tiene la finalidad de definir la estructura y la información que deberá estar contenida en el modelo de la tecnología de fabricación. Aquí es importante establecer modelos que admitan información relativa a la familia, por ejemplo, tablas o matrices que contengan información general acerca de la descripción dimensional de toda la familia, mediante el uso de variables, sin necesidad de especificarlas detalladamente en la tecnología individual.

Generación de la tecnología de fabricación de la pieza compuesta, representativa de la familia La pieza compuesta puede ser real o construida especialmente para representar al grupo de piezas y contendrá, dentro de su geometría, todo el conjunto de las diversas superficies que forman a las piezas 
que componen la familia, lo que significa que al elaborar la tecnología para la fabricación de la pieza compuesta, en principio, conllevará necesariamente a contemplar todos los pasos tecnológicos que estarán presentes en las diferentes piezas del grupo.

Generación de las tecnologías de fabricación de las piezas específicas

El modelo de tecnología para una orden de producción contendrá el procedimiento general descrito para la pieza compuesta solo con los pasos tecnológicos que aplican para el caso específico de la pieza que va a entrar en producción.

En la Figura 1 se muestra el algoritmo que se cumple en la gestión de la información contenida en la base de datos del sistema informático referida a las familias de piezas y su procesamiento para la elaboración de una tecnología específica a partir de la tecnología de la pieza compuesta.

Con la llegada de una nueva orden de producción al taller, se realiza la descripción de la pieza en su caracterización general. Puede ocurrir que la pieza haya sido procesada con anterioridad y que pertenezca a una familia ya existente, en este caso solo habrá que recuperar la información de la pieza de la base de datos y generar la tecnología de fabricación a partir de la tecnología de la pieza compuesta.

Si la pieza es procesada por primera vez, se realiza su caracterización específica mediante la asignación del valor correspondiente a cada variable. Al realizar su descripción, el sistema trata de incluirla en una familia existente, de ser posible, se procede a generar la tecnología de fabricación para la nueva pieza, a partir de la tecnología de la pieza compuesta almacenada en la base de datos.

Puede ocurrir que la nueva pieza no sea pertinente a ninguna de las familias existentes y que sea necesario elaborar completamente su tecnología por primera vez. En este caso, sería considerada como una pieza compuesta que daría inicio a una nueva familia.

En ocasiones con la llegada de nuevos miembros a una familia hay que realizar ajustes en el diseño de la pieza compuesta y en su tecnología de fabricación, para que sea completamente representativa de su familia.

\section{Desarrollo de la aplicación. Requerimientos informáticos}

Para obtener una aplicación sustentada en el procedimiento descrito, que sea lo más genérica posible para las empresas de manufactura, fueron establecidos como requerimientos informáticos de la aplicación los siguientes:

R1) Almacenar en una base de datos las características de las piezas, máquinas herramienta y utillaje tecnológico de la empresa, para utilizar esta información durante la generación de las tecnologías.

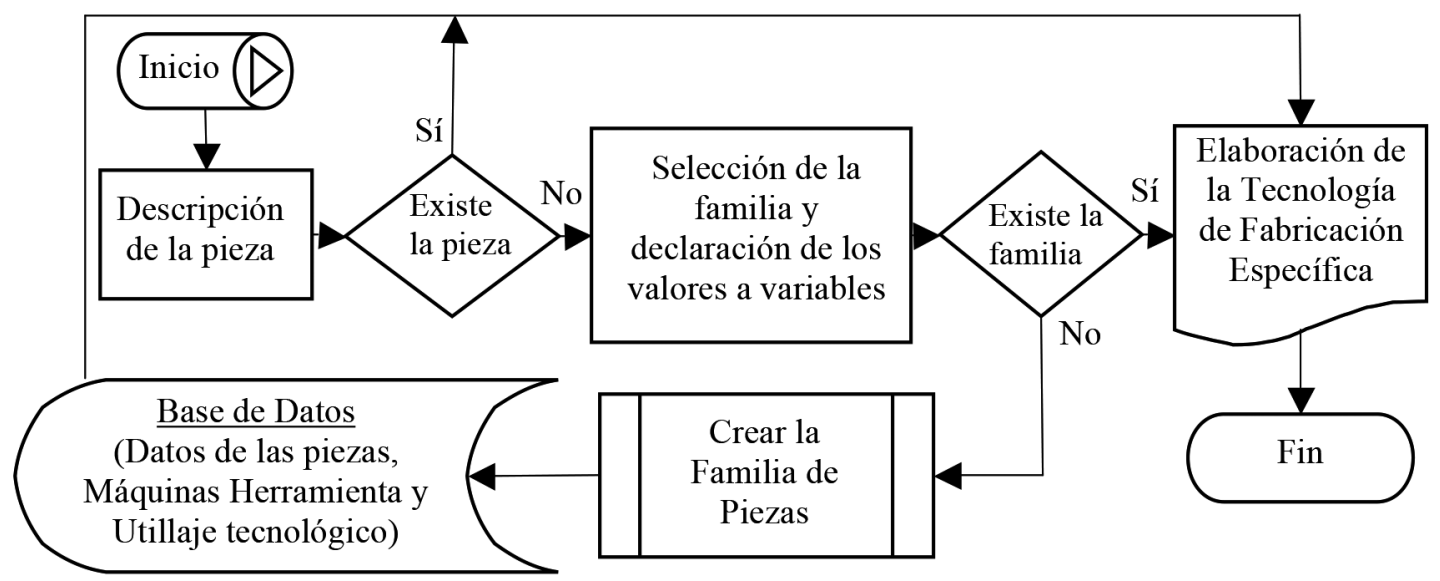

Figura 1. Algoritmo para la generación de tecnologías de maquinado mediante tecnología de grupo. 
R2) Gestionar la información de las familias de piezas mediante un sistema de codificación.

R3) Gestionar la información contenida en la tecnología de fabricación de la pieza compuesta para que sirva como modelo durante la generación de las tecnologías específicas de las piezas que pertenecen a la familia.

R4) Permitir la visualización e impresión de la tecnología generada.

La aplicación informática se desarrolló sobre plataforma libre y utiliza en la capa de datos el sistema de gestión de base de datos MySQL, en la capa de negocio los framework Symfony, para construir aplicaciones Web con PHP, y Doctrine, especializado en comunicación con bases de datos y como lenguaje de programación PHP. En la capa de presentación el framework ExtJS, especializado en creación de interfaces similares a Windows y JavaScript como lenguaje.

\section{Caso de estudio. Generación de tecnología para un tornillo}

A través del análisis de un caso de estudio se ilustra las potencialidades de dicha herramienta en la generación de la tecnología de manufactura para una familia de elementos roscados, específicamente tornillos hexagonales.

En la Tabla 1 se muestran los valores de las dimensiones normalizadas correspondientes a cada variable geométrica del diseño de los tornillos.

Tabla 1. Dimensiones normalizadas de un grupo de tornillos de cabeza hexagonal.

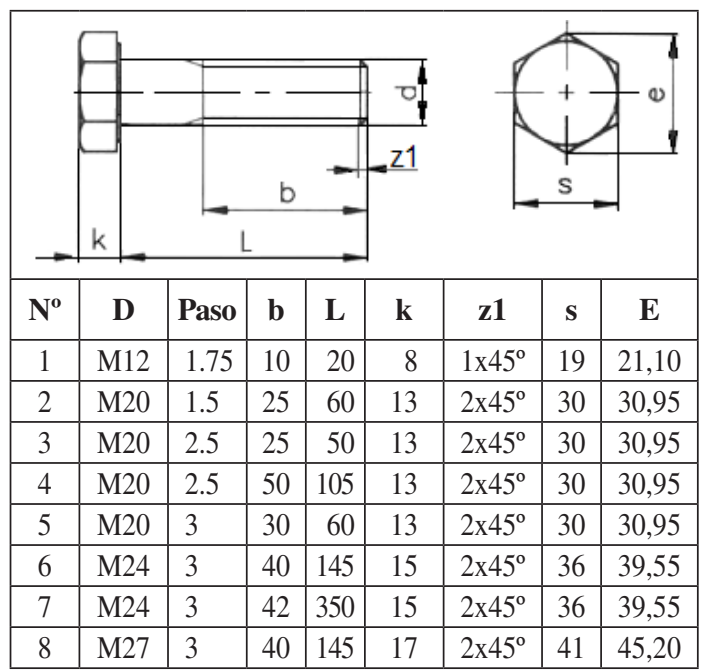

La Figura 2, representa la interface del sistema para la generación de TG en la que se destacan tres áreas esenciales:

Área 1: información sobre las piezas. (Identificación, código, plano con sus dimensiones y su material). Área 2: Información del modelo de carta de ruta tecnológica.

Área 3: Información del modelo de carta de operación tecnológica.

En el Área 1 se indica la representación gráfica de la pieza a fabricar con su geometría y dimensiones principales, la identificación de las superficies que la componen, su modelo 3D, el código y el material. En el Área 2 se ofrece información general sobre la pieza y el material en bruto así como, la descripción de la ruta tecnológica que seguirá en el taller indicándose el equipamiento tecnológico a utilizar, el tipo y cantidad de operarios involucrados, y el tiempo de ejecución de cada operación tecnológica. El Área 3 está destinada a la descripción de la operación tecnológica, indicándose la denominación de la operación, el tipo y modelo de la máquina herramienta que será utilizada, el material de la pieza y el utillaje tecnológico, el nombre del operario, la cantidad de unidades de producción a fabricar, el tiempo principal y tiempo auxiliar. Se describen las colocaciones y los pasos tecnológicos a seguir para transformar la pieza en bruto en pieza terminada con los regímenes de corte a emplear y el tiempo principal para cada paso tecnológico.

\section{RESULTADOS}

Se obtuvo un procedimiento para la generación de Tecnología de Grupo en la manufactura de piezas, y se desarrolló una herramienta informática que sirve como soporte para la implementación de dicho procedimiento.

Los resultados obtenidos muestran una vía para incrementar la eficiencia y la capacidad de respuesta de la pequeña y mediana empresa manufacturera, ante la creciente demanda de fabricación de una gran variedad de productos.

El procedimiento desarrollado para la generación de tecnologías de maquinado basado en la TG puede ser asimilado por empresas del sector manufacturero y la herramienta informática puede ser personalizada 


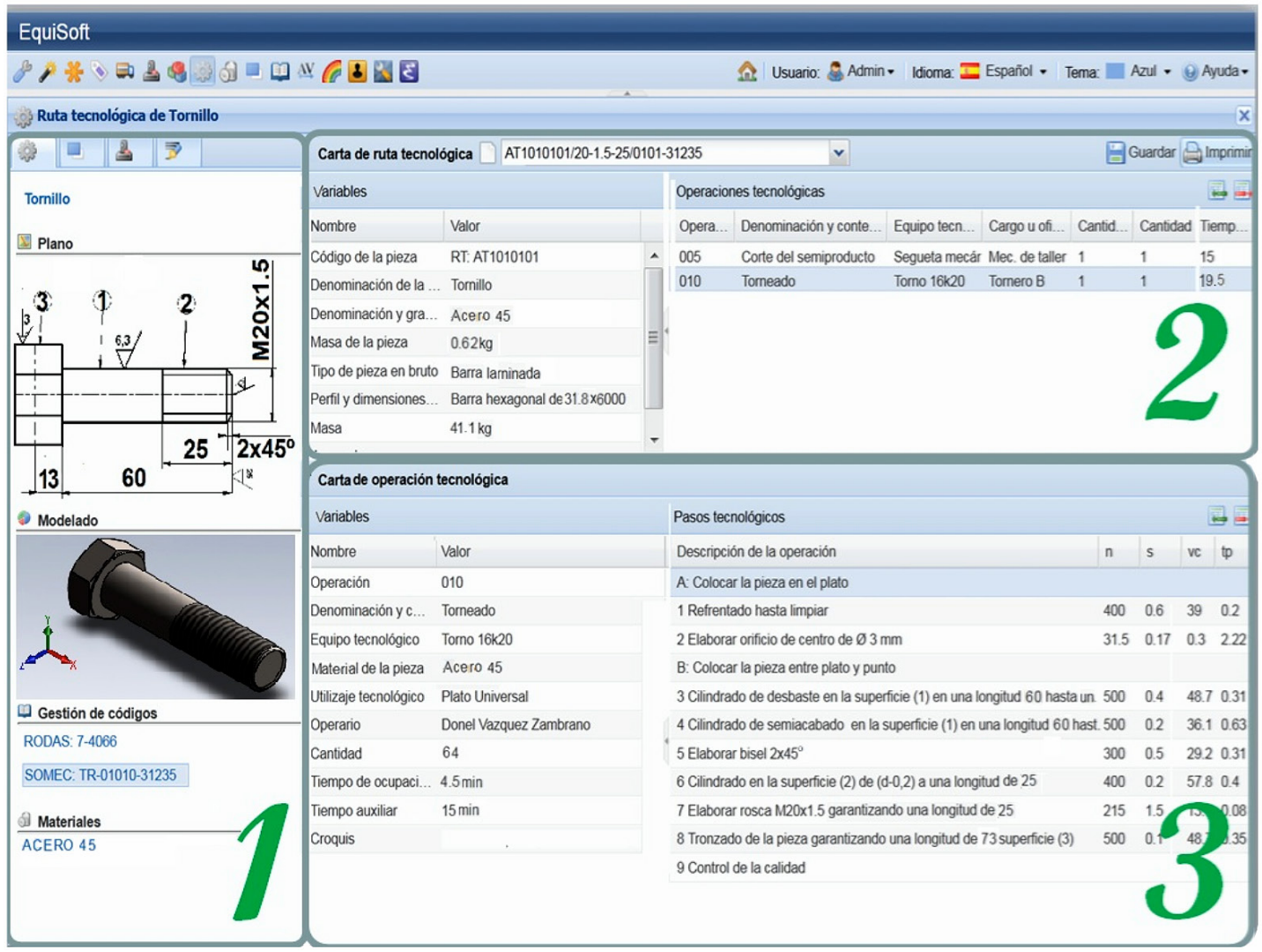

Figura 2. Interface del sistema para la generación de tecnologías de maquinado mediante tecnología de grupo.

con los formatos de documentación tecnológica específicos.

Si el volumen del trabajo de manufactura es suficientemente alto se deberán emplear técnicas de reconocimiento de piezas basadas en la Inteligencia Artificial (IA) para evitar la subjetividad en la selección o clasificación de las piezas y dinamizar así la producción.

Para el caso de empresas con volúmenes de producción medios y bajos, y con poca o mediana variedad de nomenclaturas de piezas, se recomienda realizar la clasificación inicial de las piezas mediante la participación de un especialista con experticia en los sistemas de manufactura, lo que permitirá simplificar esta tarea.

La posibilidad de generar automáticamente la tecnología para cada pieza miembro de la familia a partir de la tecnología de la pieza compuesta del grupo, le infiere a la herramienta informática desarrollada gran potencialidad, toda vez que se simplifica considerablemente la tarea de proyección tecnológica.

Con la implementación del procedimiento se podrá hacer un uso más racional del equipamiento tecnológico disponible así como, de los gastos en herramental ya que se pueden sistematizar pasos tecnológicos, herramientas y dispositivos.

\section{CONCLUSIONES}

Se plantean como conclusiones de la presente investigación las siguientes:

1. La aplicación de la tecnología de grupo favorece la organización eficiente de la producción, toda vez que permite utilizar la experiencia en la generación de tecnologías de fabricación de piezas con semejanzas geométricas y 
tecnológicas así como, la sistematización de los recursos materiales y procedimientos tecnológicos empleados.

2. La correcta selección del sistema de clasificación y codificación es un elemento indispensable para una adecuada implementación de la tecnología de grupo y estará en correspondencia con las necesidades específicas de cada empresa.

3. Se obtuvo un procedimiento para la generación de las tecnologías de fabricación de los miembros de una familia de piezas a partir de un modelo genérico con el empleo de variables, las que asumirán valores específicos en función de la pieza concreta a fabricar.

4. Se desarrolló una herramienta informática que sirve de soporte al procedimiento creado para la generación de Tecnología de Grupo.

\section{REFERENCIAS}

[1] F.S. Hsieh and C.Y. Chiang. "Workflow planning in Holonic manufacturing systems with extended contract net protocol". En: Next-Generation Applied Intelligence. Springer Berlin Heidelberg, pp. 701-710. 2009.

[2] E. Chacón and M. Indriago. "Implementación de Supervisores en una Arquitectura de Referencia Basadas en Sistemas de Manufactura Holónicos". Revista Iberoamericana de Automática e Informática Industrial RIAI. Vol. $7 \mathrm{~N}^{\mathrm{o}}$ 3, pp. 72-82. 2010.

[3] O. Roulet-Dubonnet and P. Ystgaard. "An application of the Holonic manufacturing system to a flexible assembly cell". Proceedings of the 5th international conference on industrial applications of holonic and multi-agent systems for manufacturing. Springer-Verlag Berlin, Heidelberg, pp. 29-38. 2011.

[4] J. Barbosa, P. Leitao, E. Adam and D. Trentesaux. "Dynamic self-organization in holonic multi-agent manufacturing systems: The ADACOR evolution". Computers in Industry. Vol. 66, pp. 99-111. 2015.

[5] J.A. Araúzo, R. del-Olmo-Martínez, J.J. Laviós y J.J. de-Benito-Martín. "Programación y Control de Sistemas de Fabricación Flexibles: un Enfoque Holónico". Revista Iberoamericana de Automática e Informática Industrial RIAI. Vol. $12 \mathrm{~N}^{\mathrm{o}}$ 1, pp. 58-68. 2015.
[6] J.J.L. Villahoz, R. del Olmo Martínez, A.A. Arauzo and J.M.G. Ordax. "Price updating in combinatorial actions for coordination of manufacturing multiagent systems". En Trends in Practical Applications of Agents and Multiagent Systems. Springer Berlin Heidelberg, pp. 201-207. 2010.

[7] S. Ruiz, O.D. Castrillón y W.A. Sarache. "Una Metodología Multiobjetivo para Optimizar un Ambiente Job Shop". Información Tecnológica. Vol. $23 \mathrm{~N}^{\mathrm{o}}$ 1, pp. 35-46. 2012.

[8] M. Frutos-Alazard and F. Tohmé-Hauptmann. "Técnicas evolutivas en problemas multiobjetivos en el proceso de planificación de la producción". Ingeniería Industrial. Vol. $33 \mathrm{~N}^{\circ}$ 1, pp. 50-59. 2012.

[9] S. Ruiz, O.D. Castrillón y W.A. Sarache. "Metodología selectiva de dinámica poblacional para optimizar un ambiente multiobjetivo de producción Job Shop". Revista de Matemática: Teoría y Aplicaciones. Vol. $22 \mathrm{~N}^{\circ}$ 1, pp. 113-134, 2015.

[10] S.P. Mitrofanov. "The Scientific Principles of Group Technology". Leningrad. English Translation, National Library for Science and Technology, Washington, D.C., 1966.

[11] M.V. Tatikonda and U. Wemmerlöv. "Adoption and implementation of group technology classification and coding systems: insights from seven case studies". International Journal of Production Research. Vol. 30, Issue 9, 1992.

[12] R. Thamma, E.D. Kirby, A. Ohri, P. Rinalidi y M. Rajai. "Group Technology Paves the Road for Automation". International Transaction Journal of Engineering, Management, \& Applied Sciences \& Technologies. Vol. 5 Issue 2, p. 105. 2014.

[13] R. Logendran and F. Barnard. "Sequencedependent group scheduling problems in flexible flow shops". International Journal of Production Economics. Vol. $102 \mathrm{~N}^{\mathrm{o}} 1$, pp. 66-86. 2006.

[14] A.A. Massote. "Algoritmos de tecnología de grupo para proyectos de células de manufactura". Exacta, São Paulo, Brasil. Vol. 4, pp. 31-44. 2006.

[15] A.R. Tiradentes, E. Gonçalves and A.J. Tiberti. "Algoritmo genético de agrupamento para formação de módulos de arranjo físico". Gestión de la Producción. São Carlos, Brasil. Vol. 15 No $^{\circ}$, pp. 393-405. 2008. 
[16] G. Pinot and M. Nasser. "An exact method for the best case in a group sequence: Application to a general shop problem". En 13th IFAC Symposium on Information Control Problems in Manufacturing, pp. 1269-1274. 2009.

[17] M. Zandieh, B. Dorri and A.R. Khamseh. "Robust metaheuristics for group scheduling with sequence-dependent setup times in hybrid flexible flow shops". The International Journal of Advanced Manufacturing Technology. Vol. $43 \mathrm{~N}^{\circ}$ 8, pp. 767-778. 2009.

[18] P.D. Medina, E.A. Cruz y M. Pinzón. "Generación de celdas de manufactura usando el algoritmo de ordenamiento binario (AOB)". Scientia et Technica. Vol. $1 \mathrm{~N}^{\circ}$ 44, pp. 106110. 2010.

[19] O. Cardín, N. Mebarki and G. Pinot. "A study of the robustness of the group scheduling method using an emulation of a complex FMS'. Int. J. Production Economics. Vol. 146, pp. 199-207. 2013.
[20] J.J. Bravo, J.P. Orejuela y Z. Menduiña. "Aproximación a la medición del impacto del Backorder en sistemas de manufactura flexible". Revista Facultad de Ingeniería Universidad de Antioquia. Vol. 65, pp. 99111. 2012.

[21] C. Cerda. "Propuesta de implementación de la Tecnología de Grupo (TG) para la Fábrica de Muebles VITEFAMA de la Ciudad de Cuenca, Ecuador". (Tesis de grado). 2013.

[22] P. Ortega. "Tecnología de grupos aplicada en el mecanizado de tornillos de hueso humano" (Disertación Doctoral). Universidad de Los Andes. Bogotá, Colombia. 2003.

[23] J.K. Kuric. "Development of new Classification Methods for CAPP Systems. 16th International Research Expert. Conference" Trends in the Development of Machinery and Associated Technology TMT 2012, Dubai, UAE, 10-12 September 2012. 